\title{
Self-Organizing Compact Modeling Methodology for High-Speed Passive Electrical Interconnection Structures
}

\author{
Tom Dhaene \\ University of Antwerp, Middelheimlaan 1, 2020 Antwerpen, Belgium \\ tom.dhaene@ua.ac.be \\ Agilent Technologies, EEsof Comms EDA, Lammerstraat 20, 9000 Gent, Belgium \\ tom_dhaene@agilent.com
}

\begin{abstract}
New modeling technology is developed that allows engineers to define the frequency range, layout parameters, material properties and desired accuracy for automatic generation of simulation models of general passive electrical structures. It combines electromagnetic (EM) accuracy of parameterized passive models with the simulation speed of analytical models. The adaptive algorithm doesn't require any a priori knowledge of the dynamics of the system to select an appropriate sample distribution and an appropriate model complexity. With this technology, designers no longer must put up with legacy modeling techniques or invest resources in examining new ones.
\end{abstract}

\section{Introduction}

Component and circuit models are a cornerstone of EDA (Electronic Design Automation) technology. With wireless and wireline designs constantly increasing in complexity and operating at higher frequencies, design engineers push the limits of their EDA tool's passive analytical models. Often, these passive models are used outside their operational range, causing the EDA tool to return inaccurate simulation results. The inconsistencies of legacy modeling techniques from the 1970s and 1980s hinder the accuracy of these models when applied to different processes and frequencies. Exceeding a model's frequency limit causes errors due to the model's failure to account for higher-order propagation modes. Limitations of the equivalent circuit model, such as frequency independent inductive or capacitive elements, also lead to simulation errors. Since most EDA tools do not proactively report such errors, they propagate through the design flow and may not be discovered until a prototype fails to perform as expected. To avoid errors and inconsistencies, full-wave EM simulation is required to fully characterize the structure and produce an accurate Sparameter model of the discontinuity that is then used by the circuit simulator.

Developing new models is not a trivial task! To model a single parameter over a range of values, several sample points are required. Since the model can be a function of many layout parameters (line width, length, metal thickness, dielectric constant, substrate thickness, loss tangent, etc.) there is an exponential growth in the number of samples as the number of layout parameters increases. Also, developing a new model usually requires a highly skilled person working for an extended period - several weeks or even months - to build, test and produce the desired analytical model. If the 
requirement is for a complete library of models, the total effort is multiplied by the number of models sought. This task needs to be weighed against measurement-based or EM-based modeling on a case-by-case basis.

Some common approaches to modeling issues have limiting factors [1]-[2]. Methods using pre-calculations of equivalent circuits, using a variety of look-up tables, fitting equations and interpolation techniques can have a limited number of samples and have insufficient interpolation methods. One clear example where the dependability of these techniques comes into question is with high-Q resonant circuits such as those used in narrow band filters. Using discrete data grids and interpolation techniques with such circuits might cause the generated model to suffer from either "oversampling" or "undersampling." With oversampling, too many data samples are collected and model generation is inefficient; on the other hand, with undersampling, too few data samples are collected and the model is not completely defined.

As an alternative to building classic analytical models, engineers can utilize a fullwave EM modeling tool to fully characterize a given passive component. This method permits accurate characterization of the actual passive structure to be used, accounting for higher-order mode propagation, dispersion and other parasitic effects. However, the calculation time required for full-wave EM simulation of a given component makes real-time circuit tuning impossible.

A new efficient adaptive sampling and modeling technique addresses this model accuracy dilemma. The 'Multidimensional Adaptive Parameter Sampling' algorithm (MAPS) selects a limited set of data samples in consecutive iterations, and interpolates all S-parameter data using rational and multinomial fitting models. This algorithm allows important details to be modeled by automatically sampling the response of the structure more densely where the S-parameters are changing more rapidly. The goal is minimizing the total number of samples needed, while maximizing the information provided by each new sample. The modeling technique combines the speed and flexibility of analytical models, and accuracy and generality of full-wave EM simulation in one compact parameterized passive model [3]-[4].

\section{Adaptive Modeling and Sampling Technique}

The MAPS technique builds a global fitting model of the chosen parameters, handling frequency and geometrical dependencies separately. Multidimensional polynomial (or multinomial) fitting techniques are used to model the geometrical dependencies, while rational fitting techniques [5]-[6] are used to handle frequency dependencies. The modeling process does not require any a priori knowledge of the circuit under study. Different adaptive algorithms are combined to efficiently generate a parameterized fitting model that meets the predefined accuracy. This includes the adaptive selection of an optimal number of data samples along the frequency axis and in the geometrical parameter space, and adaptive selection of the optimal order of the multinomial-fitting model.

The number of data points is selected to avoid oversampling and undersampling. The process of selecting data points and building models in an adaptive way is called 
reflective exploration [7]. Reflective exploration is useful when the process that provides the data is very costly, which is the case for full-wave EM simulators. Reflective exploration requires reflective functions that are used to select new data points. For example, the difference between two fitting models can be used as a reflective function. Also, some physical rules, such as a passivity-check, can be used as a reflective function. The modeling process starts with an initial set of data points. New data points are selected near the maximum of the reflective function until the desired accuracy is reached.

The model complexity is automatically adapted to avoid overmodeling (overshoot or ringing) and undermodeling, and the model covers the whole parameter and frequency space and can easily be used for optimization purposes.

The MAPS modeling technique follows four steps to adaptively build a model.

- Step One: The frequency response of the circuit is calculated at a number of discrete sample points (using the Agilent Momentum full-wave EM simulator [8]). The Adaptive Frequency Sampling (AFS) algorithm [6] selects a set of frequencies and builds a rational model for the S-parameters over the desired frequency range (Figure 1).

- Step Two: A multinomial is fitted to the S-parameter data at multiple discrete frequencies (Figure 2).

- Step Three: This model is written as a weighted sum of orthonormal multinomials. The multinomials only depend on the layout parameters. The weighting coefficients preceding the orthonormal multinomials in the sum are only frequency dependent (Figure 3).

- Step Four: Using the AFS models built in step one, the coefficients can be calculated over the whole frequency range (Figure 4). These coefficients, together with the orthonormal multinomials, are stored in a database for use during extraction afterwards.

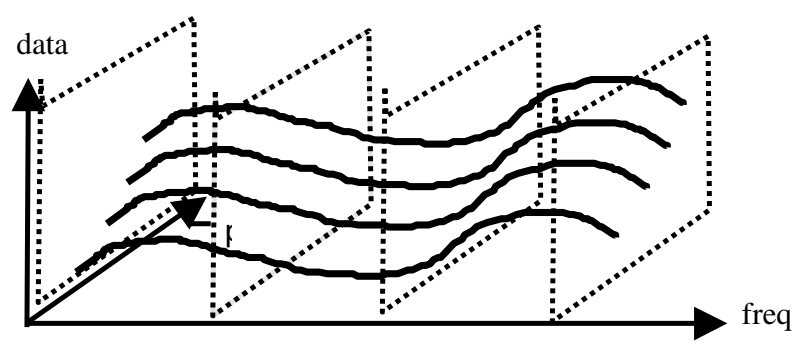

Fig. 1. Step One: AFS rational models over the desired frequency range, derived from fullwave EM simulation. 


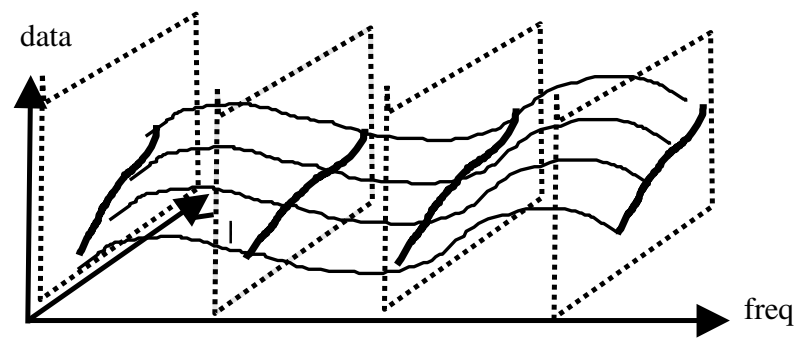

Fig. 2. Step Two: Multinomial models are created at discrete frequencies.

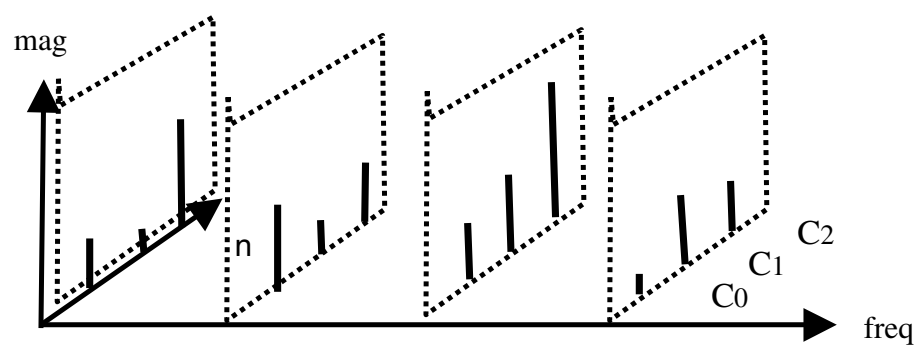

Fig. 3. Step Three: Creation of the coefficients of orthogonal multinomials at discrete frequencies.

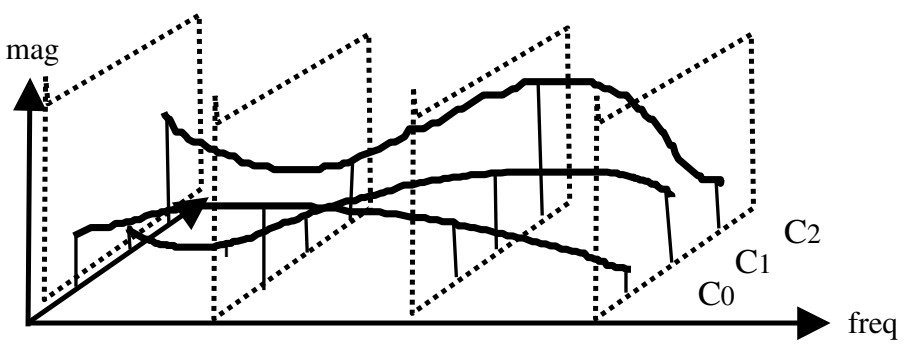

Fig. 4. Step Four: Calculation of coefficients of orthogonal multinomials over the entire frequency range.

\section{Example}

The automated modeling technique was used to generate analytical circuit models for all sub-parts (transmission line, open end, slot coupler, step in width, corner-fed 
patch) of a slot-coupled microstrip-fed patch antenna structure (figure 5). This modeling step is a one-time, up-front time investment. A double sided duroid substrate was used (thickness $=31 \mathrm{mil} \& 15 \mathrm{mil}, \varepsilon_{\mathrm{r}}=2.33, \operatorname{tg} \delta=0.0012$ ).

First, parameterized circuit models were built for all substructures of the circuit. For example, the corner-fed patch (figure 6) circuit model was built over the following parameter range (table 1):

Table 1. Parameter ranges of corner-fed patch

\begin{tabular}{|c|c|c|}
\hline variable & min & max \\
\hline L_patch & 320 & 400 \\
& mil & mil \\
W_feed & $5 \mathrm{mil}$ & $30 \mathrm{mil}$ \\
$\mathrm{f}$ & & \\
& $5 \mathrm{GHz}$ & 15 \\
& & $\mathrm{GHz}$ \\
\hline
\end{tabular}

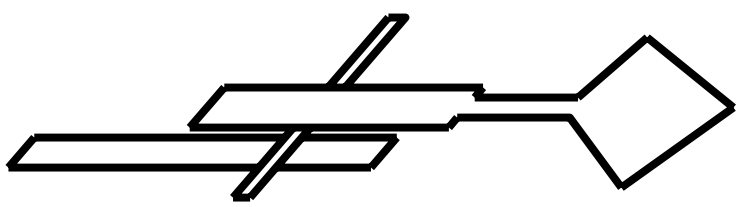

Fig. 5. Slot-coupled microstrip-fed patch antenna structure

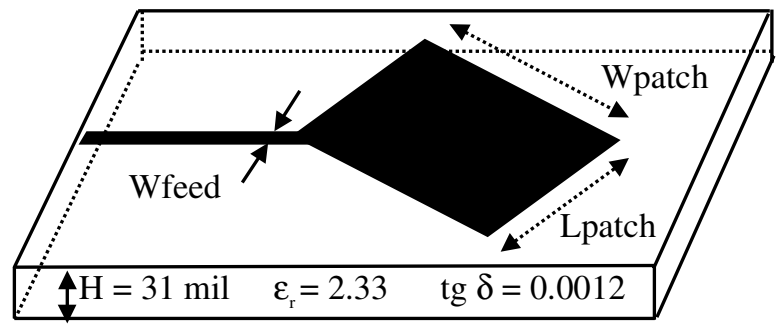

Fig. 6. Layout of corner-fed patch

The automated modeling tool (ADS Model Composer) selected 25 data points (= discrete layouts) in an adaptive way, and grouped all S-parameter data all in one global, compact, analytical model. ADS Momentum was used as planar EM simulator [8]. The desired accuracy level was set to $-55 \mathrm{~dB}$. In figure 7 , the reflection coefficient $\mathrm{S}_{11}$ of the corner-fed patch is shown as a function of frequency and width.

Then, the parameterized circuit models were used to simulate the overall antenna structure (figure 5). Figure 8 shows $S_{11}$ simulated with Momentum, and with the new analytical circuit models for all sub-components (divide and conquer approach). Both results correspond very well. However, the simulations based on the circuit models 
easily allow optimization and tuning, and took only a fraction of the time of the full-wave simulation ( 2 seconds compared to 96 minutes on a $450 \mathrm{MHz}$ Pentium II computer).

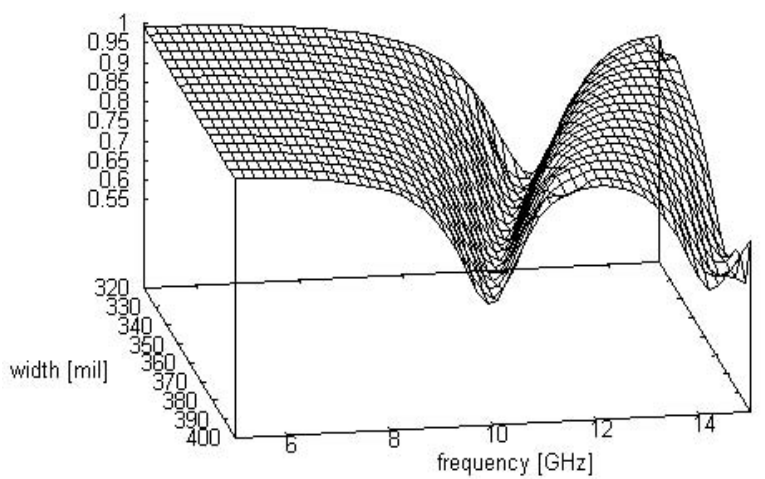

Fig. 7. Reflection coefficient $S_{11}$ of corner-fed patch (W_feed = 8 mil)

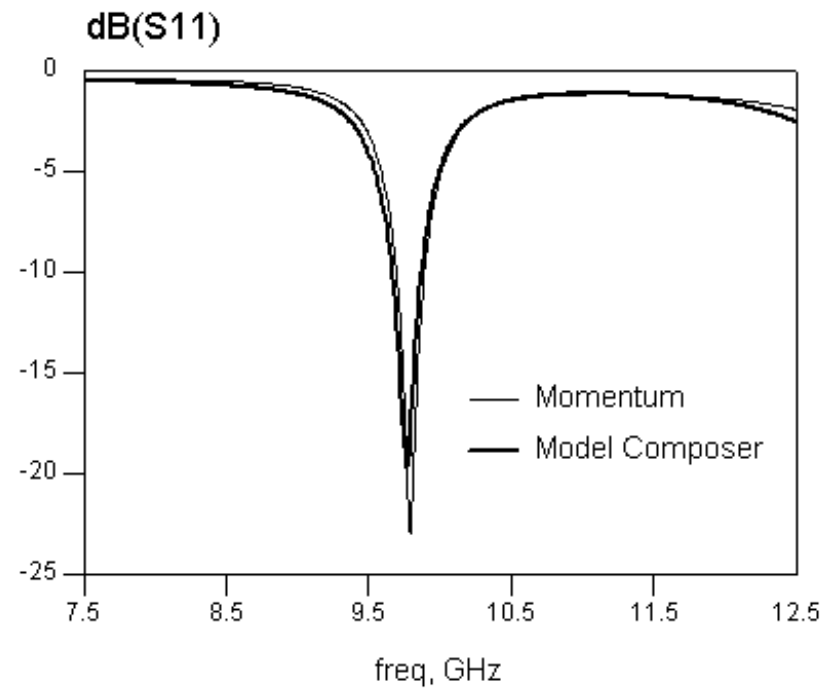

Fig. 8. Reflection coefficient $S_{11}$ of slot-coupled microstrip-fed patch antenna

\section{Conclusions}

An advanced modeling technique was presented for building parameterized models for general passive microwave and RF structures. The models are based on full-wave EM simulations, and have a user-defined accuracy. Once generated, the analytical 
models can be grouped in a library, and incorporated in an EDA tool where they can be used for simulation, design and optimization purposes. A patch antenna example was given to illustrate the technique. The results based on the parameterized models correspond very well with the global full-wave simulations. However, the time required for a simulation using the compact analytical circuit models was only a fraction of the time required for a global full-wave simulation.

\section{References}

1. Chaki S., Aono S., Andoh N., Sasaki Y., Tanino N., Ishihara O.: Experimental Study on Spiral Inductors, Proceedings of the IEEE Symposium on Microwave Theory and Techniques, (1995) 753-756.

2. Liang J-F., Zaki K. A.: CAD of Microwave Junctions by Polynomial Curve Fitting, Proceedings of the IEEE Symposium on Microwave Theory and Techniques, (1993) 451454.

3. De Geest J., Dhaene T., Fache N., De Zutter D.: Adaptive CAD-Model Building Algorithm for General Planar Microwave Structures, IEEE Transactions on Microwave Theory and Techniques, vol. 47, no. 9, (1999) 1801-1809.

4. Dhaene T., De Geest J., De Zutter D.: EM-based Multidimensional Parameterized Modeling of General Passive Planar Components, Proceedings of the IEEE Symposium on Microwave Theory and Techniques, Vol. 3, (2001) 1745-1748.

5. Dhaene, T., Ureel, J., Fache, N., De Zutter, D.: Adaptive Frequency Sampling Algorithm for Fast and Accurate S-parameter Modeling of General Planar Structures, Proceedings of the IEEE Symposium on Microwave Theory and Techniques, (1995) 1427-1430.

6. Dhaene, T.: Automated Fitting and Rational Modeling Algorithm for EM-Based SParameter, Proceedings of the Applied Parallel Computing Conference, LNCS 2367, (2002), pp. 99-105.

7. U. Beyer and F. Smieja, Data Exploration with Reflective Adaptive Models, Computational Statistics and Data Analysis, vol. 22, (1996) 193-211.

8. Momentum software, Agilent EEsof Comms EDA, Agilent Technologies, Santa Rosa, CA. 